\title{
From CDMS to SuperCDMS
}

\section{P. C. F. Di Stefano*t and Y. Ricci}

Department of Physics, Queen's University, Kingston, ON, Canada, K7L 3N6

E-mail: distefan@queensu.ca

The Cryogenic Dark Matter Search (CDMS) and SuperCDMS experiments seek hypothetical weakly-interacting, massive particles (WIMPs) that could account for the majority of matter in the Universe, by means of cryogenic germanium detectors. We provide an update on the status of the SuperCDMS experiment, and discuss the joint CDMS-EDELWEISS analysis.

The 2011 Europhysics Conference on High Energy Physics-HEP 2011,

July 21-27, 2011

Grenoble, Rhône-Alpes France

*Speaker.

${ }^{\dagger}$ On behalf of the CDMS collaboration. 


\section{CDMS}

The problem of dark matter has been around since 1933 [1]: most of the matter in the Universe only appears through its gravitational interactions. Particle physics may provide a solution to this problem in the guise of weakly interacting, massive particles (WIMPs) [2]. Efforts have been underway to detect WIMPs directly since the mid 1980s. The experimental challenge is great, since the average energy deposits are only expected to range up to a few tens of $\mathrm{keV}$, and since the expected rate of events is far below usual levels of background radiation. The Cryogenic Dark Matter Search (CDMS) collaboration has deployed various semi-counducting cryogenic detectors for over a decade. The current phase of the experiment is located in the Soudan Underground Laboratory, beneath a depth of rock equivalent to $2.1 \mathrm{~km}$ of water, providing substantial shielding against cosmogenic backgrounds. The detectors are operated at a temperature of the order of $50 \mathrm{mK}$, and measurement of phonons using tungsten transition-edge-sensors provides the energy deposited in the detector by a particle interaction. A simultaneous measurement of charge allows to distinguish between the highly ionizing dominant radioactive background coming from electrons and gammas, and the nuclear recoil signal that would be caused by WIMPs. Though the neutron background also creates nuclear recoils, the mean free path of fast neutrons in the detectors is of the order of a centimeter, whereas that of WIMPs would be greater than light years, providing a tool to distinguish and understand this contribution to the background. The phonon channel also provides some spatial resolution in the detector, allowing to reject some of the surface events that cold be misidentified as signal otherwise.

CDMS has deployed up to thirty individual detector modules, each of mass up to some $230 \mathrm{~g}$. The most recent data set has a threshold of $5 \mathrm{keV}$, and an effective net exposure of up to $379 \mathrm{~kg}$.d. Four candidate events remain in the data, with an expected background contribution of two events with a significant uncertainty [3, 4]. Limits have been extracted on WIMP couplings to matter using the optimal interval technique [5]. Given the usual assumptions on the particle physics models and astrophysical halo parameters, there is some amount of disagreement between these limits and recent possible observations of low-mass WIMPs by the CRESST experiment [9].

\section{The joint CDMS-EDELWEISS analysis}

The EDELWEISS collaboration uses germanium detectors that are similar to those of CDMS. Before final analysis of the most recent EDELWEISS data set [7], both collaborations agreed to jointly develop methods to combine their data [6] and they settled on one a priori. The sensitivities of CDMS and EDELWEISS to WIMPs are shown in Fig. 1. They are similar at high masses since the experiments have similar exposures. At low masses, CDMS is more sensitive because of its lower threshold. Constraints on WIMPs from various experiments, including the combined CDMS-EDELWEISS limit [8], are shown in Fig. 2.

\section{Status of SuperCDMS}

The SuperCDMS collaboration will improve on the sensitivity of CDMS, first in the current experimental setup at Soudan, then in the deeper SNOLAB site [10]. Fifteen novel iZIP detectors [11], amounting to a total mass of $9.5 \mathrm{~kg}$, have been installed at Soudan as of November 2011. 


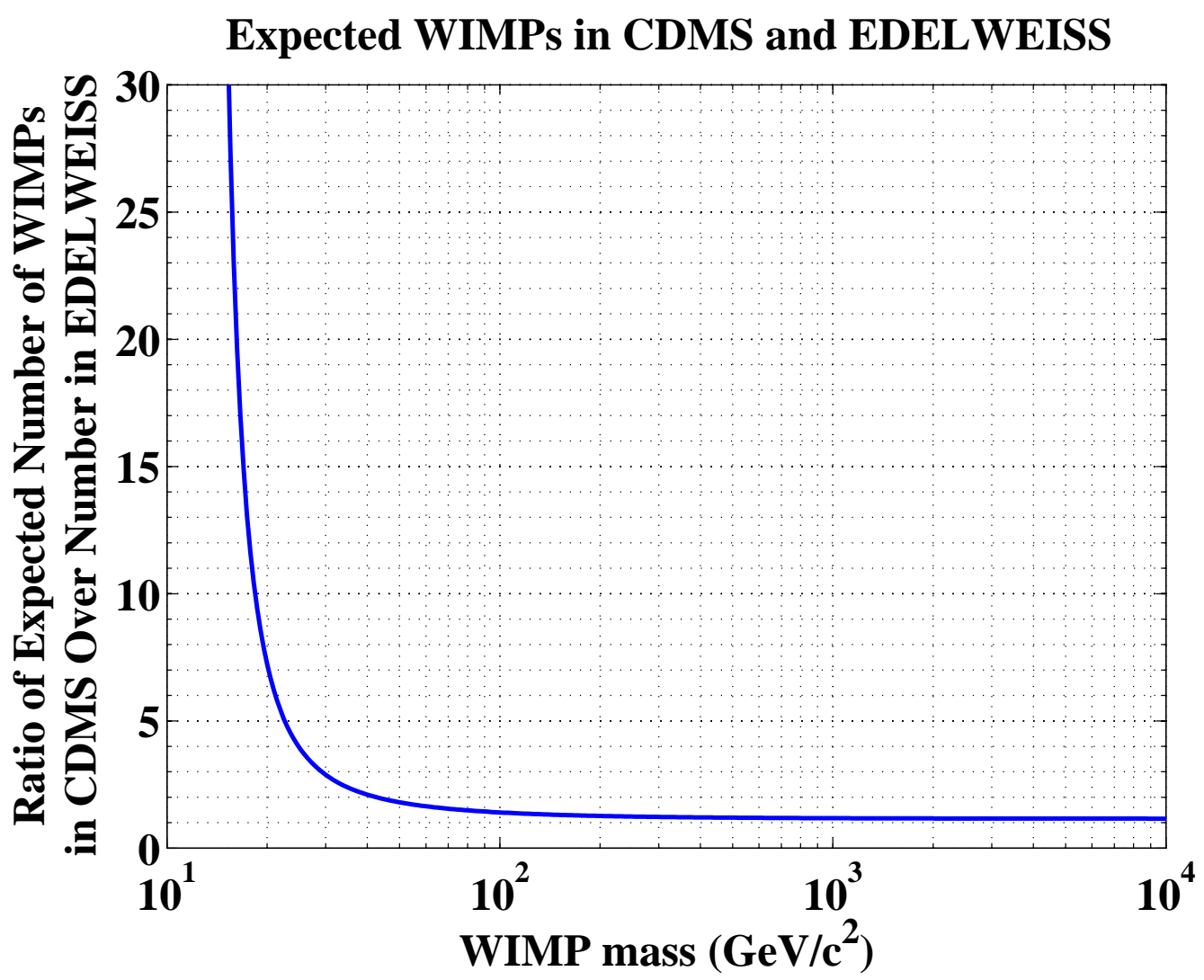

Figure 1: Ratio of number of WIMP events expected in CDMS [3] over that expected in EDELWEISS [7]. At high WIMP masses, the ratio tends to a value close to one, since the exposures are similar. At low WIMP masses, the lower threshold of CDMS makes it more sensitive to WIMPs. Astrophysical and nuclear parameters are the same as in [8].

They weigh $600 \mathrm{~g}$ each, and use interleaved electrodes creating an inhomogenous electric field to improve identification of surface events. Demonstrated rejection of surface events is better than $3: 10^{5}$. The target sensitivity for Soudan is $5 \times 10^{-45} \mathrm{~cm}^{2}$ with no background; the target sensitivity for the SNOLAB phase is $3 \times 10^{-46} \mathrm{~cm}^{2}$ with no background.

\section{References}

[1] F. Zwicky, Helv. Phys. Acta 6110 (1933).

[2] G. Bertone, Particle Dark Matter: Observations, Models and Searches, Cambridge University Press, 2010.

[3] CDMS Collaboration, Science 3271619 (2010).

[4] CDMS Collaboration, Phys. Rev. Lett. 102011301 (2009).

[5] S. Yellin, Phys. Rev. D 66032005 (2002).

[6] S. Yellin, (2011) [arXiv:1105.2928].

[7] EDELWEISS Collaboration, Phys. Lett. B 702329 (2011) [arXiv: 1103 . 4070]. 
90\% CL Limits: Simple Merger of CDMS and EDELWEISS Data

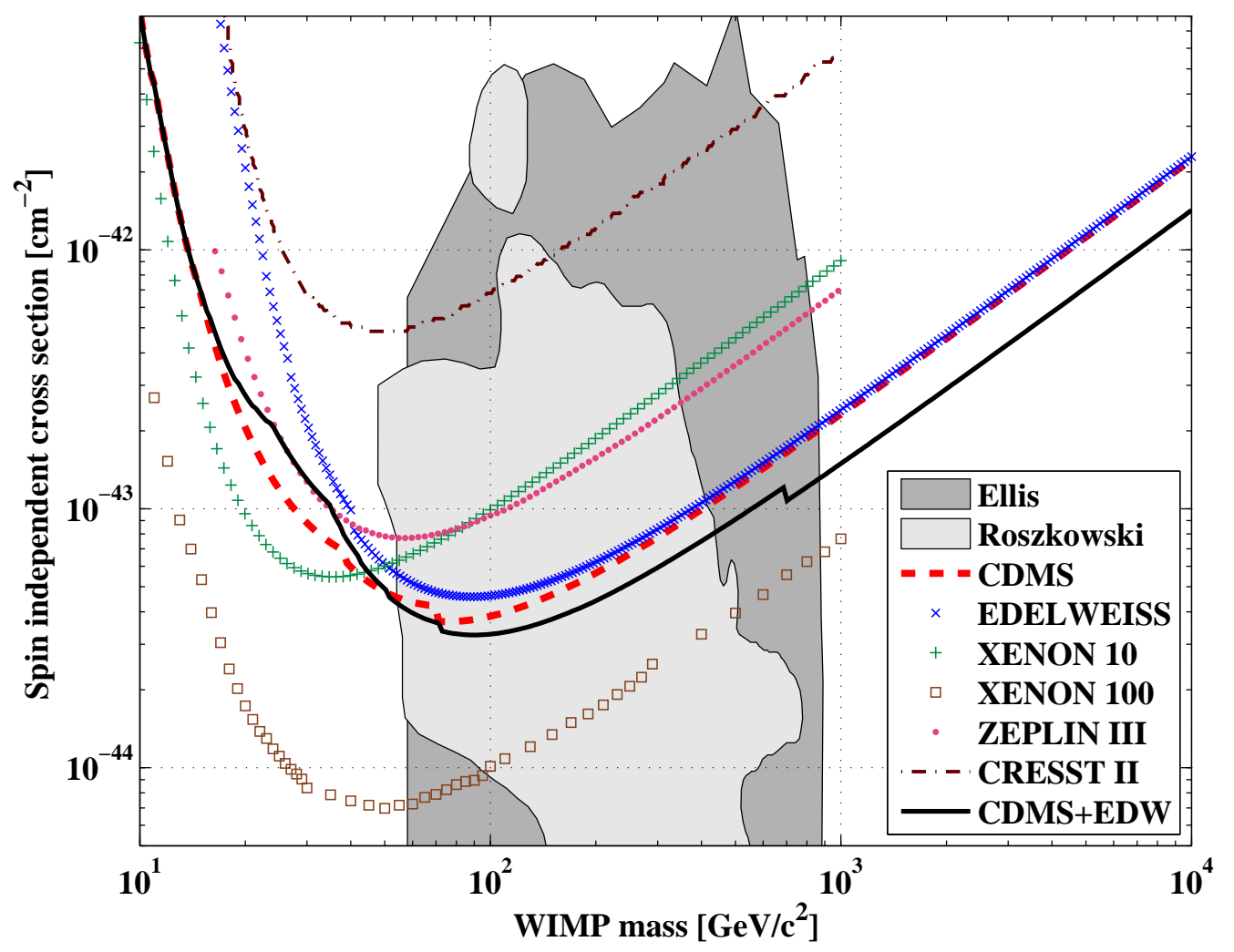

Figure 2: Limits on WIMPs, from [8]. The combined CDMS-EDELWEISS limit [8] improves over the best of the two individual limits $[3,7]$ by up to a factor 1.6 at certain masses, but is worse at low masses. Other data sets from $[12,13,14,15,16,17]$

[8] CDMS and EDELWEISS Collaborations, Phys. Rev. D 84 011102(R) (2011) [arXiv:1105.3377v2].

[9] CRESST Collaboration, (2011) [arXiv:1109.0702v1].

[10] www.snolab.ca

[11] M. Pyle et al, AIP Conf. Proc. (LTD13) 1185223 (2009).

[12] XENON 10 Collaboration, Phys. Rev. C 79045807 (2009).

[13] J. Ellis et al, Phys. Rev. D 71095007 (2005).

[14] L. Roszkowski et al, J. High Energy Phys. 075 (2007).

[15] XENON100 Collaboration, Phys. Rev. Lett. 107131302 (2011).

[16] CRESST II Collaboration, Astrop. Phys. 31270 (2009).

[17] ZEPLIN-III Collaboration, Phys. Rev. D 80052010 (2009). 